\title{
On the mass and momentum transport in the Navier-Stokes slip layer
}

\author{
Janusz Badur • Michał Karcz • Marcin Lemański
}

Received: 25 October 2010/Accepted: 18 April 2011/Published online: 17 May 2011

(C) The Author(s) 2011. This article is published with open access at Springerlink.com

\begin{abstract}
The Navier-Stokes slip boundary conditions are considered as conditions following from the mass and momentum balances within a thin, shell-like moving boundary layer. A problem of consistency between different models, that describes the internal and external friction in a viscous fluid, is stated within the framework of a proper form of the layer momentum balance. Appropriate constitutive equations for friction forces are formulated. The common features of the Navier, Stokes, Reynolds, and Maxwell concepts of a boundary slip layer are revalorized and discussed. Different mobility mechanisms connected with the transpiration phenomena, important for flows in micro- and nanochannels, are classified as a part of equations for the external friction.
\end{abstract}

keywords Slip velocity - Navier-Stokes layer .

Navier number $\cdot$ Mobility coefficients

\section{Introduction}

The applicability of the Navier-Stokes slip boundary conditions to the study of many micro- and nanoscale devices has been demonstrated in the literature using continuum models and Molecular Dynamics simulations, but there have been limited experimental investigations

\footnotetext{
J. Badur $\cdot$ M. Karcz $(\bowtie) \cdot$ M. Lemański

Energy Conversion Department, Institute of Fluid Flow

Machinery, Polish Academy of Sciences, Gdańsk, Poland

e-mail: michal.karcz@imp.gda.pl

J. Badur

e-mail: janusz.badur@imp.gda.pl

M. Lemański

e-mail: marcin.lemanski@imp.gda.pl
}

concerning the effects of the external friction. This is primarily due to the difficulty of obtaining accurate experimental results at small scales. Many experimentalists attempt to model a fluid at small scales via the study of a very viscous fluid at macro scale. It corresponds to a fluid system with a low Reynolds number (in the bulk) and a low Navier number (near the surface) which is typical for small-scale fluid flow (Karniadakis et al. 2005; Ho and Tai 1998; Gad-El-Hak 2001).

However, experimental techniques only partially take into account properties of fluid being in contact with the wall surface at small scales, and do not take into account the properties of the solid. Some analytical solutions of the benchmark problems like: the Poiseuille flow, steady Couette flow, a pendulum ball in the oscillating motion, steadystate behavior of a fluid between two parallel walls one of which is oscillating, etc. (To et al. 2010; Matthews and Hill 2009), are required for obtaining an accurate experimental results. Having an analytical patterns, one can determine not only the applicability of the proposed slip models but also the value of the external friction coefficient, or its dimensionless counterpart- the Navier number. Therefore, in the literature there are many articles (see You et al. 2007; Lauga and Stone 2003), where the problem has been studied, also within the continuum framework, using the Navier-Stokes equations with the Navier-Stokes boundary conditions at the fluid-solid interface(s). For instance, the flow of a fluid near an oscillating wall with slip has been studied in detail by Matthews and Hill (2009).

From the present literature, it can be concluded that the approach to nano- or microchannel flows, could be further based on the same classical Navier-Stokes equations in the bulk. Its application, however, is limited to the use of postulate wall-fluid friction models with various parameters that should be determined either empirically, or by comparisons 
with independent simulations performed with more exact molecular models. The wall-gas friction models become even more complex when the intermittent transition from a constant to the rate dependent Navier number is observed, and Knudsen regimes of flow are considered (Thompson and Trojan 1997; Zhu and Granick 2001; Priezjev 2007). In the present article, we study a consistency of the Navier-Stokes model of fluid in the bulk domain as well as in the boundary slip layer. In some contradiction to the presented in the literature "second order slip velocity", as in Karniadakis et al. (2005), we propose to generalize the Navier-Stokes slip layer by adding a layer momentum transport. It leads to determination of slip velocity $v_{\mathrm{s}}$ on the base of two-dimensional differential equations instead of algebraic relations. Thus, for a proper definition of layer momentum vector we need also a proper definition of surface mass density $\rho_{\mathrm{s}}$. Therefore, a layer balance of mass is added to the basic set of layer governing equations. The article is organized as follows. In the following section, we describe details of kinematics of middle surface of the layer. The balances of the layer mass and momentum are introduced in Sects. 3 and 4. Constitutive relations for the quantities of layer are briefly summarized in Sect. 5. Details of Navier, Stokes, Maxwell, and Reynolds slip layers are presented in Sect. 6, where different mobility mechanisms are also being formulated. The summary and conclusions are given in the last section.

\section{Moving shell-like region in a fluid continuum}

In our treatment of a problem of generalization of the Navier-Stokes boundary slip layer (denoted as $\mathcal{M}^{+} \mathcal{M}^{-}$), we shall assume that this layer can be treated as some shelllike thin domain moving in a space with a geometrical, migration velocity $\mathbf{w}$. This shell-like domain divides the continuum into a continuum $\mathcal{A}$, that is a fluid under consideration, and a continuum $\mathcal{B}$ which can be a free surface, solid body, or a second fluid. If both $\mathcal{A}$ and $\mathcal{B}$ are fluids then the Navier-Stokes boundary layer represents the moving interfacial region, where physical properties change in a radical manner. For instance, in a thin transition layer between liquid and vapor, the change of density is so steep, that it looks like a jump throughout the layer thickness. Therefore, we assume that in the layer we observe so-called "apparent" material properties, quite different than in the bulk of continua $\mathcal{A}$ and $\mathcal{B}$. Thus, we define an excess of layer density $\rho_{\mathrm{s}}\left[\mathrm{kg} \mathrm{m}^{-2}\right]$, the layer particle velocity $\mathbf{v}_{\mathrm{s}}\left[\mathrm{ms}^{-1}\right]$, an excess of layer momentum density $\rho_{\mathrm{s}} \mathbf{v}_{\mathrm{s}}$, and a surface excess of momentum flux $\mathbf{p}_{\mathrm{s}}$, (Badur and Karcz 2010). In general, this shell-like region moves with the geometrical velocity $\mathbf{w}$ which differs from material velocity $\mathbf{v}_{A}$ in $\mathcal{A}$, velocity $\mathbf{v}_{B}$ in $\mathcal{B}$ and velocity $\mathbf{v}_{\mathrm{s}}$ in $\mathcal{M}^{+} \mathcal{M}^{-}$. In particular case, the velocity $\mathbf{w}$ denotes the rate of change of phase transition surface within the fluid being at rest (Scriven 1960; dell'Isola and Kosiński 1993). In most technically interesting cases, the component $w_{n}$ normal to moving middle surface $\mathcal{M}$, differs from normal components of $\mathbf{v}_{A}, \mathbf{v}_{B}$ and $\mathbf{v}_{\mathrm{s}}$. It practically means that we have also some mass transport across the layer. Indeed, the geometrical velocity field is not a priori known and usually is determined from a special evolution equation (Scriven 1960). If $\mathbf{w}=\mathbf{v}_{\mathrm{s}}$ then the moving layer is material, if $\mathbf{w}=$ $\mathbf{v}_{\mathrm{s}} \mathbf{I}_{\mathrm{s}}+w_{n} \mathbf{n}$ then the surface is semi-coherent.

Note, that, in difference to Navier and Stokes, who assumed that the surface layer density is equal to zero, we want to determine the slip velocity $\mathbf{v}_{\mathrm{s}}$ from an independent balance of the layer momentum. In some cases, it simplifies to the well-known balance of the boundary traction forces. In the case of immiscible liquids being in contact, the tangential components $\mathbf{v}_{\mathrm{S}} \mathbf{I}_{\mathrm{s}}$ can be approximately described to be $\frac{1}{2}\left(\mathbf{v}_{A}+\mathbf{v}_{B}\right) \mathbf{I}_{\mathrm{s}}$. Quite similarly, only in a special case there is $\rho_{\mathrm{s}}=\frac{1}{2}\left(\rho_{A}+\rho_{B}\right) h$, where $h$ is a finite thickness of the layer. We are based here on a general surface kinematics elaborated by Pietraszkiewicz (1977) and Stumpf and Badur (1993). The general form of the surface balances of mass, momentum, angular momentum, energy, and entropy, etc. is given by Zmitrowicz (1987), Slattery et al. (2007), and dell'Isola and Kosiński (1993). Within the Navier-Stokes layer, we introduce a new concept of an "excess of momentum flux" which can be described by the surface symmetrical diade $\mathbf{p}_{\mathrm{s}}$. It is responsible for momentum transport within the layer, therefore, it has tangential and normal components. If $\mathbf{a}_{\alpha}, \mathbf{n}(\alpha=1,2)$ are the base vectors on the middle surface of the layer $\mathcal{M}$, then, we postulate the surface momentum flux in a following form:

$\mathbf{p}_{\mathrm{s}}(\xi)=p^{\alpha \beta} \mathbf{a}_{\alpha} \otimes \mathbf{a}_{\beta}+p^{n \alpha} \mathbf{n} \otimes \mathbf{a}_{\alpha}+p^{\alpha n} \mathbf{a}_{\alpha} \otimes \mathbf{n}+p^{n n} \mathbf{n} \otimes \mathbf{n}$,

where $\xi^{\alpha}, \alpha=1,2$ are local surface curvilinear coordinates on $\mathcal{M}$. Physical properties of the layer are unknown a priori since they depend on the resulting apparent properties in both continua $\mathcal{A}$ and $\mathcal{B}$. For instance, elastic recoverable properties of $\mathbf{p}_{\mathrm{s}}$ depend on an actual shape of the surface $\mathcal{M}$, mainly from its stretching and bending. Many authors, especially those using Molecular Dynamics for modeling of the layer behavior, postulate that due to strong induced elasticity of the fluid layer, it changes from the elastic fluid (only recoverable spherical deformations) into an elastic fluid with recoverable shape deformations (To et al. 2010). Similarly, owing to induced strong anisotropy, the internal viscosity of the fluid layer can be described by four apparent viscosity coefficients (Goodrich 1981), and the anisotropic friction by four coefficients of the friction tensor (Zmitrowicz 1987). 
Having postulated three basic notions $\rho_{\mathrm{s}}, \mathbf{v}_{\mathrm{s}}, \mathbf{p}_{\mathrm{s}}$, let us now recall a few mathematical relations required for establishing of balance of the layer mass and momentum. Let us first introduce the Weatherburn surface fundamental diades (Stumpf and Badur 1993):

$$
\begin{aligned}
& \mathbf{I}_{\mathrm{s}}=\mathbf{I}-\mathbf{n} \otimes \mathbf{n}=\operatorname{grad}_{\mathrm{s}} \mathbf{x}_{\mathrm{s}}=a^{\alpha \beta} \mathbf{a}_{\alpha} \otimes \mathbf{a}_{\beta}, \\
& \mathbf{I I}_{\mathrm{s}}=-\operatorname{grad}_{\mathrm{s}} \mathbf{n}=b^{\alpha \beta} \mathbf{a}_{\alpha} \otimes \mathbf{a}_{\beta},
\end{aligned}
$$

called the first and second fundamental form of the surface $\mathcal{M}$. Surface gradient acts also on the coordinate dependent base $\mathbf{a}_{\alpha}, \mathbf{n}$, therefore, the surface gradient of velocity is calculated to be:

$$
\begin{aligned}
\operatorname{grad}_{\mathrm{s}} \mathbf{v}_{\mathrm{s}} & =\left(v^{\alpha} \mathbf{a}_{\alpha}+v_{n} \mathbf{n}\right) \otimes \nabla_{\beta} \mathbf{a}^{\beta} \\
& =\left(v_{\alpha \mid \beta}-v_{n} b_{\alpha \beta}\right) \mathbf{a}^{\alpha} \otimes \mathbf{a}^{\beta}+\left(v^{\alpha} b_{\alpha \beta}+v_{n, \beta}\right) \mathbf{n} \otimes \mathbf{a}^{\beta},
\end{aligned}
$$

and the surface divergence of this vector is:

$$
\begin{aligned}
\operatorname{div}_{\mathrm{s}} \mathbf{v}_{\mathbf{s}}=\mathbf{C}_{1,2} \operatorname{grad}_{\mathrm{s}} \mathbf{v}_{\mathbf{s}} & =\left(v_{\alpha \mid \beta}-v_{n} b_{\alpha \beta}\right) a^{\alpha \beta} \\
& =v^{\alpha}{ }_{\mid \alpha}-v_{n} b^{\alpha}{ }_{\alpha}=\operatorname{div}_{\mathbf{s}}\left(\mathbf{v}_{\mathrm{s} \|}\right)-v_{n} \mathrm{I}_{b},
\end{aligned}
$$

where the invariants of the second fundamental form or the curvature diade are: $\mathrm{I}_{b}=\operatorname{tr} \mathbf{I I}_{\mathrm{s}}=b^{\alpha}{ }_{\alpha}=b^{1}{ }_{1}+b^{2}{ }_{2}=\left(\frac{1}{r_{1}}+\right.$ $\left.\frac{1}{r_{2}}\right), \mathrm{II}_{b}=\operatorname{det} \mathbf{I I}_{\mathrm{s}}=\operatorname{det}\left(b_{\alpha \beta}\right)$. Rate of surface deformation, in analogy to the three-dimensional case, is defined as a symmetric part of the surface gradient of velocity, (Stumpf and Badur 1993):

$$
\begin{aligned}
\mathbf{d}_{\mathrm{s}}= & \frac{1}{2}\left(\operatorname{grad}_{\mathrm{s}} \mathbf{v}_{\mathrm{s}}+\operatorname{grad}_{\mathrm{s}}^{\mathrm{T}} \mathbf{v}_{\mathrm{s}}\right) \\
= & {\left[\frac{1}{2}\left(v_{\alpha \mid \beta}+v_{\beta \mid \alpha}\right)-v_{n} b_{\alpha \beta}\right] \mathbf{a}^{\alpha} \otimes \mathbf{a}^{\beta} } \\
& +\frac{1}{2}\left(v^{\alpha} b_{\alpha \beta}+v_{n, \beta}\right)\left(\mathbf{n} \otimes \mathbf{a}^{\beta}+\mathbf{a}^{\beta} \otimes \mathbf{n}\right),
\end{aligned}
$$

and in analogy to $3 \mathrm{D}$, the first invariant of $\mathbf{d}_{\mathrm{s}}$ is:

$$
\mathrm{I}_{\mathbf{d}_{\mathrm{s}}}=\operatorname{tr} \mathbf{d}_{\mathrm{s}}=\mathbf{C}_{1,2} \mathbf{d}_{\mathrm{s}}=v^{\alpha}{ }_{\mid \alpha}-v_{n} \mathrm{I}_{b} .
$$

Quite similarly, the surface gradient of the flux of momentum is:

$$
\begin{aligned}
\operatorname{grad}_{\mathrm{s}} \mathbf{p}= & \mathbf{p}_{\mathrm{s}} \otimes\left(\nabla_{\gamma} \mathbf{a}^{\gamma}\right)=p^{\alpha \beta}{ }_{\mid \gamma} \mathbf{a}_{\alpha} \otimes \mathbf{a}_{\beta} \otimes \mathbf{a}^{\gamma} \\
& +p^{\alpha \beta} b_{\alpha \gamma} \mathbf{n} \otimes \mathbf{a}_{\beta} \otimes \mathbf{a}^{\gamma}+p^{\alpha \beta} b_{\beta \gamma} \mathbf{a}_{\alpha} \otimes \mathbf{n} \otimes \mathbf{a}^{\gamma} \\
& +p^{n \alpha}{ }_{\mid \gamma}\left(\mathbf{n} \otimes \mathbf{a}_{\alpha} \otimes \mathbf{a}^{\gamma}+\mathbf{a}_{\alpha} \otimes \mathbf{n} \otimes \mathbf{a}^{\gamma}\right) \\
& +\left(2 p^{n \alpha} b_{\alpha \gamma}+p^{n n}{ }_{\mid \gamma}\right) \mathbf{n} \otimes \mathbf{n} \otimes \mathbf{a}^{\gamma} \\
& -p^{n \alpha} b^{\varepsilon}{ }_{\gamma}\left(\mathbf{a}_{\varepsilon} \otimes \mathbf{a}_{\alpha} \otimes \mathbf{a}^{\gamma}+\mathbf{a}_{\alpha} \otimes \mathbf{a}_{\varepsilon} \otimes \mathbf{a}^{\gamma}\right) \\
& -p^{n n} b^{\varepsilon}{ }_{\gamma}\left(\mathbf{a}_{\varepsilon} \otimes \mathbf{n} \otimes \mathbf{a}^{\gamma}+\mathbf{n} \otimes \mathbf{a}_{\varepsilon} \otimes \mathbf{a}^{\gamma}\right),
\end{aligned}
$$

and its divergence:

$$
\begin{aligned}
\operatorname{div}_{\mathrm{s}} \mathbf{p}_{\mathrm{s}}=\mathbf{C}_{2,3} \operatorname{grad}_{\mathrm{s}} \mathbf{p}_{\mathrm{s}}= & \left(p^{\alpha \beta}{ }_{\mid \beta}-p^{n \beta} b^{\alpha}{ }_{\beta}-\mathrm{I}_{b} p^{\alpha n}\right) \mathbf{a}_{\alpha} \\
& +\left(p^{\alpha \beta} b_{\alpha \beta}+p^{n \alpha}{ }_{\mid \alpha}-\mathrm{I}_{b} p^{n n}\right) \mathbf{n} .
\end{aligned}
$$

Let us recall the useful identities $\left(d_{t}=\mathrm{d} / \mathrm{d} t ; \partial_{t}=\partial /\right.$ $\left.\partial t ; \partial_{n}=\partial / \partial n\right)$ :

- the Reynolds transport theorem for mass in continuum $\mathcal{A}$ with the moving subsurface $S^{+} \subset \mathcal{M}^{+}$:

$d_{t} \int_{A} \rho_{A} \mathrm{~d} v=\int_{A} \partial_{t} \rho_{A} \mathrm{~d} v+\int_{\partial A} \rho_{A} \mathbf{v}_{A} \cdot \mathbf{n}_{A} \mathrm{~d} s+\int_{S^{+}} \rho_{A} \mathbf{w} \cdot \mathbf{n}^{+} \mathrm{d} s$,

which after using of 3D Green-Ostrogradzki theorem:

$$
\int_{A} \operatorname{div}\left(\rho_{A} \mathbf{v}_{A}\right) \mathrm{d} v=\int_{\partial A} \rho_{A} \mathbf{v}_{A} \cdot \mathbf{n}_{A} \mathrm{~d} s+\int_{S^{+}} \rho_{A} \mathbf{v}_{A} \cdot \mathbf{n}^{+} \mathrm{d} s,
$$

takes the following form:

$$
\begin{aligned}
d_{t} \int_{A} \rho_{A} \mathrm{~d} v= & \int_{A}\left[\partial_{t} \rho_{A}+\operatorname{div}\left(\rho_{A} \mathbf{v}_{A}\right)\right] \mathrm{d} v \\
& +\int_{S^{+}} \rho_{A}\left(\mathbf{w}-\mathbf{v}_{A}\right) \cdot \mathbf{n}^{+} \mathrm{d} s
\end{aligned}
$$

- the Slattery transport theorem for the surface mass (Zmitrowicz 1987; Slattery et al. 2007):

$d_{t} \int_{S} \rho_{\mathrm{s}} \mathrm{d} s=\int_{\substack{S \\ \cdot \mathbf{n}_{l} \mathrm{~d} l}}\left(\partial_{t} \rho_{\mathrm{s}}-\mathrm{I}_{b} w_{n} \rho_{\mathrm{s}}\right) \mathrm{d} s+\int_{\partial S} \rho_{\mathrm{s}} \mathbf{v}_{\mathrm{s} \|} \cdot \mathbf{n}_{l} \mathrm{~d} l+\int_{l} \rho_{\mathrm{s}} \mathbf{w}_{\|}$

together with 2D Green-Ostrogradzki identity:

$$
\int_{S} \operatorname{div}\left(\rho_{\mathrm{s}} \mathbf{v}_{\mathrm{s} \|}\right) \mathrm{d} s=\int_{\partial S} \rho_{\mathrm{s}} \mathbf{v}_{\mathrm{s} \|} \cdot \mathbf{n}_{l} \mathrm{~d} l+\int_{l} \rho_{\mathrm{s}} \mathbf{v}_{\mathrm{s} \|} \cdot \mathbf{n}_{l} \mathrm{~d} l,
$$

takes the form:

$$
\begin{aligned}
d_{t} \int_{S} \rho_{\mathrm{s}} \mathrm{d} s= & \int_{S}\left[\partial_{t} \rho_{\mathrm{s}}+\operatorname{div}\left(\rho_{\mathrm{s}} \mathbf{v}_{\mathrm{s} \|}\right)-\mathrm{I}_{b} w_{n} \rho_{\mathrm{s}}\right] \mathrm{d} s \\
& +\int_{l} \rho_{\mathrm{s}}\left(\mathbf{w}_{\|}-\mathbf{v}_{\mathrm{s} \|}\right) \cdot \mathbf{n}_{l} \mathrm{~d} l,
\end{aligned}
$$

where $\mathbf{w}_{\|}=\mathbf{w I}_{\mathrm{s}}$ and $\mathbf{v}_{\mathrm{s} \|}=\mathbf{v} \mathbf{I}_{\mathrm{s}}$ are tangential velocities.

In quite analogous manner, repeating this reasoning for the momentum vectors, we obtain:

- the Reynolds transport theorem for momentum in continuum $\mathcal{A}$ with the moving surface $S^{+}$:

$$
\begin{aligned}
& d_{t} \int_{A} \rho_{A} \mathbf{v}_{A} \mathrm{~d} v=\int_{A} \partial_{t}\left(\rho_{A} \mathbf{v}_{A}\right) \mathrm{d} v+\int_{\partial A} \rho_{A} \mathbf{v}_{A} \otimes \mathbf{v}_{A} \cdot \mathbf{n}_{A} \mathrm{~d} s \\
& \quad+\int_{S^{+}} \rho_{A} \mathbf{v}_{A} \otimes \mathbf{w} \cdot \mathbf{n}^{+} \mathrm{d} s=\int_{A}\left[\partial_{t}\left(\rho_{A} \mathbf{v}_{A}\right)+\operatorname{div}\left(\rho_{A} \mathbf{v}_{A} \otimes \mathbf{v}_{A}\right)\right] \mathrm{d} v \\
& \quad+\int_{S^{+}} \rho_{A} \mathbf{v}_{A} \otimes\left(\mathbf{w}-\mathbf{v}_{A}\right) \cdot \mathbf{n}^{+} \mathrm{d} s
\end{aligned}
$$


- the Slattery transport theorem for the surface momentum (Zmitrowicz 1987; Slattery et al. 2007):

$$
\begin{aligned}
d_{t} \int_{S} \rho_{\mathrm{s}} \mathbf{v}_{\mathrm{s}} \mathrm{d} s= & \int_{S}\left[\partial_{t}\left(\rho_{\mathrm{s}} \mathbf{v}_{\mathrm{s}}\right)-\rho_{\mathrm{s}} \mathbf{v}_{\mathrm{s}} w_{n} \mathrm{I}_{b}\right] \mathrm{d} s \\
& +\int_{\partial S} \rho_{\mathrm{s}} \mathbf{v}_{\mathrm{s}} \otimes \mathbf{v}_{\mathrm{s} \|} \cdot \mathbf{n}_{l} \mathrm{~d} l+\int_{l} \rho_{\mathrm{s}} \mathbf{v}_{\mathrm{s}} \otimes \mathbf{w}_{\|} \cdot \mathbf{n}_{l} \mathrm{~d} l,
\end{aligned}
$$

or

$$
\begin{aligned}
& d_{t} \int_{S} \rho_{\mathrm{s}} \mathbf{v}_{\mathrm{s}} \mathrm{d} s=\int_{S}\left[\partial_{t}\left(\rho_{\mathrm{s}} \mathbf{v}_{\mathrm{s}}\right)+\operatorname{div}_{\mathrm{s}}\left(\rho_{\mathrm{s}} \mathbf{v}_{\mathrm{s}} \otimes \mathbf{v}_{\mathrm{s} \|}\right)\right. \\
& \left.\quad-\rho_{\mathrm{s}} \mathbf{v}_{\mathrm{s}} w_{n} \mathrm{I}_{b}\right] \mathrm{d} s+\int_{l} \rho_{\mathrm{s}} \mathbf{v}_{\mathrm{s}} \otimes\left(\mathbf{w}_{\|}-\mathbf{v}_{\mathrm{s} \|}\right) \cdot \mathbf{n}_{l} \mathrm{~d} l .
\end{aligned}
$$

Let us note that the infinitesimal domain, is quite different for 3D balance and for a layer balance. In the case of $3 \mathrm{D}$ balance, it is sufficient to take the infinitesimal Euler-Cauchy cube $\mathrm{d} v=\mathrm{d} x \mathrm{~d} y \mathrm{~d} z$. On the other hand, in a layer we must cut off a part of layer, which, according to Fried and Gurtin (2005), we shall call a "pillbox" with an infinitesimal volume $\mathrm{d} v=h \mathrm{~d} S^{+} \times \mathrm{d} S^{-}$. According to the Fried-Gurtin rule of the pillbox balance, we distinguish the integration over the surfaces $S^{+}$and $S^{-}$from an integration over $\mathrm{d} a \cong \mathrm{d} h \mathrm{~d} l$ surface with $\mathbf{n}_{l}(h) \approx \mathbf{n}_{l}$ (see Fig. 1) ( dell'Isola and Kosiński 1993). Looking for a Green-like transformation for this curved surface, and bearing in mind that $\mathbf{p}_{\mathrm{s}}$ has the form (1), we obtain:

$$
\begin{gathered}
\int_{l}\left[\int_{h} \mathbf{p}_{\mathrm{s}}(h, l) \mathbf{n}_{l}(l) \mathrm{d} h\right] \mathrm{d} l \cong \int_{l}\left[\int_{h^{-}}^{h^{+}} \mathbf{p}_{\mathrm{s}}(h, l) \mathbf{n}_{l}(l)\right] \mathrm{d} l \\
\quad=\int_{l} \mathbf{p}_{\mathrm{s}} \mathbf{n}_{l} \mathrm{~d} l=\int_{S}\left[\operatorname{div}_{\mathrm{s}} \mathbf{p}_{\mathrm{s}}+\left(\partial_{\mathrm{n}} \mathbf{p}_{\mathrm{s}} \mathbf{n}\right)\right] \mathrm{d} s .
\end{gathered}
$$

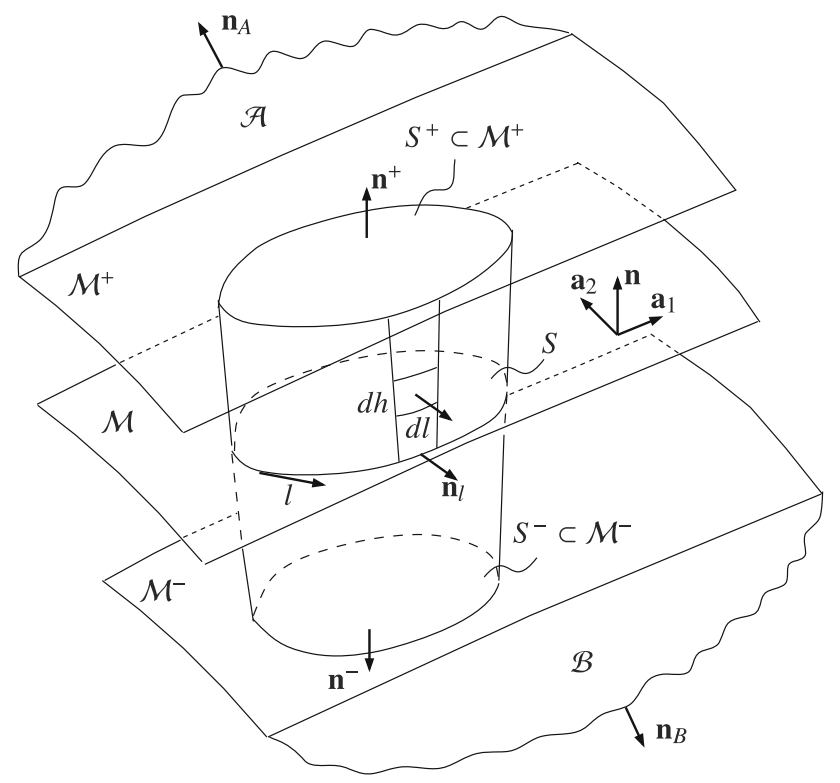

Fig. 1 Outline of the Navier-Stokes slip boundary layer
It means that the normal change of $\mathbf{p}_{\mathrm{s}}$ deals only with the outer surface components:

$\mathbf{p}_{\mathrm{s}}=\mathbf{p}_{\mathrm{s}} \mathbf{I}_{\mathrm{s}}+\left(\mathbf{p}_{\mathrm{s}} \mathbf{n}\right) \otimes \mathbf{n}$.

\section{Balance of mass in the Navier-Stokes layer}

Mass of the Navier-Stokes layer in any case cannot be taken to be constant. Rate of mass change is noticeable and cannot be simply omitted. Such phenomena as drainage, wetting, dewetting, adherence, surface nucleation, catalytic reactions, lubrication, and frequently appear in the nature and technology. Therefore, in the mathematical model of a generalized Navier-Stokes boundary slip layer, we decide to add an additional governing equation, namely a balance of the surface mass. Since the layer mass cannot be taken from "nothing", we assume that sources of mass are the bulk fluid continuum $\mathcal{A}$ and the body $\mathcal{B}$, or the material line $\mathcal{L}$ that bounds the surface $\mathcal{M}$. Thus, the mass balance of the whole system is:

$$
\begin{aligned}
d_{t} & {\left[\int_{A} \rho_{A} \mathrm{~d} v+\int_{B} \rho_{B} \mathrm{~d} v+\int_{S} \rho_{\mathrm{s}} \mathrm{d} s\right] } \\
& +\int_{S^{+}} \dot{m}_{A} \mathrm{~d} s+\int_{S^{-}} \dot{m}_{B} \mathrm{~d} s+\int_{S} \dot{m}_{S} \mathrm{~d} s+\int_{l} \dot{m}_{l} \mathrm{~d} l=0,
\end{aligned}
$$

where $\dot{m}_{A}$ and $\dot{m}_{B}$ are mass influxes $\left[\mathrm{kg} \mathrm{s}^{-1} \mathrm{~m}^{-2}\right]$ that come from the continua $\mathcal{A}$ and $\mathcal{B}$ via the surface $S^{+}$and $S^{-}$, respectively, and $\dot{m}_{l}$ is mass outflux $\left[\mathrm{kg} \mathrm{s}^{-1} \mathrm{~m}^{-1}\right]$. There are some restrictions in balances, if we separate the system onto three sub-systems divided by surfaces $S^{+}$and $S^{-}$such that:

$d_{t} \int_{A} \rho_{A} \mathrm{~d} v+\int_{S^{+}} \dot{m}_{A} \mathrm{~d} s=0 \quad$ in $\mathcal{A} \cup S^{+}$,

$d_{t} \int_{B} \rho_{B} \mathrm{~d} v+\int_{S^{-}} \dot{m}_{B} \mathrm{~d} s=0 \quad$ in $\quad \mathcal{B} \cup S^{-}$,

$d_{t} \int_{S} \rho_{\mathrm{s}} \mathrm{d} s+\int_{S} \dot{m}_{s} \mathrm{~d} s+\int_{l} \dot{m}_{l} \mathrm{~d} l=0 \quad$ on $\quad \mathcal{M} \cup \mathcal{L}$.

Thus, balancing only the mass crossing surfaces $S^{+}$and $S^{-}$we have a restriction:

$\int_{S^{+}} \dot{m}_{A} \mathrm{~d} s+\int_{S^{-}} \dot{m}_{B} \mathrm{~d} s+\int_{S} \dot{m}_{S} \mathrm{~d} s=0$.

Omitting small differences between $S^{+}, S^{-}$and $S$ we can simply write:

$\dot{m}_{A}+\dot{m}_{B}+\dot{m}_{\mathrm{s}}=0$.

It means that the layer mass has a source $\dot{m}_{\mathrm{s}}=$ $-\left(\dot{m}_{A}+\dot{m}_{B}\right)$ that is equal to contribution coming from $\mathcal{A}$ and $\mathcal{B}$. Taking the Reynolds and Slattery transport theorems (10-15) we can obtain from (21) that: 


$$
\begin{aligned}
\int_{A} & {\left[\partial_{t} \rho_{A}+\operatorname{div}\left(\rho_{A} \mathbf{v}_{A}\right)\right] \mathrm{d} v+\int_{B}\left[\partial_{t} \rho_{B}+\operatorname{div}\left(\rho_{B} \mathbf{v}_{B}\right)\right] \mathrm{d} v } \\
& +\int_{S^{+}}\left[\dot{m}_{A}+\rho_{A}\left(\mathbf{w}-\mathbf{v}_{A}\right) \cdot \mathbf{n}^{+}\right] \mathrm{d} s \\
& +\int_{S^{-}}\left[\dot{m}_{B}+\rho_{B}\left(\mathbf{w}-\mathbf{v}_{B}\right) \cdot \mathbf{n}^{-}\right] \mathrm{d} s \\
& +\int_{S}\left[\partial_{t} \rho_{\mathrm{s}}+\operatorname{div}_{\mathrm{s}}\left(\rho_{\mathrm{s}} \mathbf{v}_{\mathrm{s} \|}\right)-w_{n} \rho_{\mathrm{s}} \mathrm{I}_{\mathrm{b}}+\underline{\mathrm{m}}_{\mathrm{s}}\right] \mathrm{d} s \\
& +\int_{l}\left[\dot{m}_{l}+\rho_{\mathrm{s}}\left(\mathbf{w}_{\|}-\mathbf{v}_{\mathrm{s} \|}\right)\right] \cdot \mathbf{n} \mathrm{d} l=0 .
\end{aligned}
$$

These are precise equations of mass balance in the system under consideration. Locally, these read to be:

$\partial_{t} \rho+\operatorname{div}(\rho \mathbf{v})=0$ for $\mathcal{A} \cup \mathcal{B}$,

$\partial_{t} \rho_{\mathrm{s}}+\operatorname{div}_{\mathrm{s}}\left(\rho_{\mathrm{s}} \mathbf{v}_{\mathbf{s} \|}\right)-w_{n} \rho_{\mathrm{s}} \mathrm{I}_{b}=\dot{m}_{A}+\dot{m}_{B} \quad$ on $\quad \mathcal{M}$.

Additionally, from the balances on surfaces $S^{+}, S^{-}$and open part of boundary line $\mathcal{L}$ we obtain accompanying definitions of mass fluxes:

$\begin{array}{lll}\dot{m}_{A}=-\rho_{A}\left(\mathbf{w}-\mathbf{v}_{A}\right) \cdot \mathbf{n}^{+} & \text {on } & S^{+}, \\ \dot{m}_{B}=-\rho_{B}\left(\mathbf{w}-\mathbf{v}_{B}\right) \cdot \mathbf{n}^{-} & \text {on } & S^{-}, \\ \dot{m}_{\mathrm{s}}=-\rho_{\mathrm{s}}\left(\mathbf{w}_{\|}-\mathbf{v}_{\mathrm{s} \|}\right) \cdot \mathbf{n}_{l} & \text { on } & \mathcal{L} .\end{array}$

\section{Balance of momentum of the Navier-Stokes layer}

Great novelty of the Navier-Stokes layer is an idea of common treatment of the external friction phenomena as a true Newtonian "vis impressa", which first time in continuum mechanics can legally take part in the balance of momentum. Therefore, postulated by Navier, the friction force $\mathbf{f}_{S A}$ (on the surface $S^{+}$) and $\mathbf{f}_{S B}$ (on the surface $S^{-}$) explicitly appear in the balance of system forces. It is a crucial point of our reasoning. Both $\mathbf{f}_{S A}$ and $\mathbf{f}_{S B}$ depend on material properties of fluids $\mathcal{A}, \mathcal{B}$ and on apparent, operative properties of the layer $\mathcal{M}^{+} \mathcal{M}^{-}$. Let the system contains two continua $\mathcal{A}, \mathcal{B}$ and the pillbox of the Navier-Stokes layer bounded by material line $\mathcal{L}$. Then, the condition of a conservation of the total momentum can be written as:

$$
\begin{aligned}
d_{t} & {\left[\int_{A} \rho_{A} \mathbf{v}_{A} \mathrm{~d} v+\int_{B} \rho_{B} \mathbf{v}_{B} \mathrm{~d} v+\int_{S} \rho_{\mathrm{s}} \mathbf{v}_{\mathrm{s}} \mathrm{d} s\right] } \\
& +\int_{A \cup B}\left(\rho_{A}+\rho_{B}\right) \mathbf{b} \mathrm{d} s+\int_{S} \rho_{\mathrm{s}} \mathbf{b}_{s} \mathrm{~d} s \\
& +\int_{S^{+}}\left(\mathbf{p}_{n A}+\dot{\mathbf{r}}_{A}\right) \mathrm{d} s+\int_{S^{-}}\left(\mathbf{p}_{n B}+\dot{\mathbf{r}}_{B}\right) \mathrm{d} s \\
& +\int_{\partial A} \mathbf{p}_{n A} \mathrm{~d} s+\int_{\partial B} \mathbf{p}_{n B} \mathrm{~d} s \\
& +\int_{S}\left(\mathbf{p}_{S^{+}}+\mathbf{p}_{S^{-}}+\dot{\mathbf{r}}_{\mathrm{s}}\right) \mathrm{d} s+\int_{l}\left(\mathbf{p}_{n l}-\dot{\mathbf{r}}_{l}\right) \mathrm{d} l \\
& +\int_{\partial S} \mathbf{p}_{n l} \mathrm{~d} l=0 .
\end{aligned}
$$

Here, $\dot{\mathbf{r}}_{A}, \dot{\mathbf{r}}_{B}$, and $\dot{\mathbf{r}}_{\mathrm{s}}$ are the momentum carried with the mass flux $\dot{m}_{A}, \dot{m}_{B}, \dot{m}_{\mathrm{s}}$, respectively. Next, the traction forces on the contact surfaces are defined to be $\mathbf{p}_{n A}=\mathbf{p}_{A} \mathbf{n}^{+}$and $\mathbf{p}_{n B}=\mathbf{p}_{B} \mathbf{n}^{-}$what means that these depend on the stresses $\mathbf{p}_{A}$ and $\mathbf{p}_{B}$ in the bulk continua. Additionally, $\mathbf{p}_{S^{+}}$and $\mathbf{p}_{S^{-}}$ are two contact forces which act on the layer side $S^{+}$and $S^{-}$, respectively. On the boundary of the Fried-Gurtin pillbox there is the surface traction $\mathbf{p}_{n l}=\mathbf{p}_{\mathrm{s}} \mathbf{n}_{l}$, where $\mathbf{n}_{l}$ is a normal vector to the surface element $\mathrm{d} h \mathrm{~d} l$. If we have the surface mass flow-out $\dot{m}_{l}$ through the line $\mathcal{L}$ then additional momentum $\dot{\mathbf{r}}_{l}$ is carried out from the system. The forces $\mathbf{b}$ and $\mathbf{b}_{\mathrm{s}}$ are the conventional body forces.

Taking the physical relation that restricts momentum transport through the surface $\mathcal{M}$ :

$$
\begin{aligned}
& \int_{S^{+}} \dot{\mathbf{r}}_{A} \mathrm{~d} s+\int_{S^{-}} \dot{\mathbf{r}}_{B} \mathrm{~d} s+\int_{S} \dot{\mathbf{r}}_{\mathrm{s}} \mathrm{d} s=\int_{S}\left(\dot{\mathbf{r}}_{A}+\dot{\mathbf{r}}_{B}+\dot{\mathbf{r}}_{\mathrm{s}}\right) \mathrm{d} s \\
& \longrightarrow \quad \dot{\mathbf{r}}_{\mathrm{s}}=-\dot{\mathbf{r}}_{A}-\dot{\mathbf{r}}_{B},
\end{aligned}
$$

and assuming that the contact forces are restricted by an internal equilibrium, being some continuum realization of the Newtonian law saying on "action and reaction", we have:

$\int_{S^{+}} \mathbf{p}_{n A} \mathrm{~d} s+\int_{S^{-}} \mathbf{p}_{n B} \mathrm{~d} s+\int_{S}\left(\mathbf{p}_{S^{+}}+\mathbf{p}_{S^{-}}\right) \mathrm{d} s=0$.

Assuming further that, not already defined contact forces $\mathbf{p}_{S^{+}}, \mathbf{p}_{S^{-}}$depend on the traction forces coming from the continua $\mathcal{A}, \mathcal{B}$ and friction forces $\mathbf{f}_{S A}$ and $\mathbf{f}_{S B}$, we have within the contact surfaces, according to the law of "equality of action and reaction", the following relation:

$$
\left\{\begin{array}{l}
\mathbf{p}_{n A}+\mathbf{f}_{S A}=\mathbf{p}_{S^{+}} \\
\mathbf{p}_{n B}+\mathbf{f}_{S B}=\mathbf{p}_{S^{-}}
\end{array}\right.
$$

Using the above relations (34-36) and (16-18), we obtain:

$$
\begin{aligned}
\int_{A} & {\left[\partial_{t}\left(\rho_{A} \mathbf{v}_{A}\right)+\operatorname{div}\left(\rho_{A} \mathbf{v}_{A} \otimes \mathbf{v}_{A}\right)+\operatorname{div} \mathbf{p}_{A}-\rho_{A} \mathbf{b}\right] \mathrm{d} v } \\
& +\int_{B}\left[\partial_{t}\left(\rho_{B} \mathbf{v}_{B}\right)+\operatorname{div}\left(\rho_{B} \mathbf{v}_{B} \otimes \mathbf{v}_{B}\right)+\operatorname{div} \mathbf{p}_{B}-\rho_{B} \mathbf{b}\right] \mathrm{d} v \\
& +\int_{S}\left[\partial_{t}\left(\rho_{\mathrm{s}} \mathbf{v}_{\mathrm{s}}\right)+\operatorname{div}_{\mathrm{s}}\left(\rho_{\mathrm{s}} \mathbf{v}_{\mathrm{s}} \otimes \mathbf{v}_{\mathrm{s} \|}\right)-w_{n} \rho_{\mathrm{s}} \mathbf{v}_{\mathrm{s}}+\operatorname{div}_{\mathrm{s}} \mathbf{p}_{\mathrm{s}}\right. \\
& \left.+\partial_{n}\left(\mathbf{p}_{\mathrm{s}} \mathbf{n}\right)+\left(\mathbf{p}_{n A}+\mathbf{p}_{n B}+\mathbf{f}_{S A}+\mathbf{f}_{S B}\right)-\left(\rho_{\mathrm{s}} \mathbf{b}_{\mathrm{s}}+\dot{\mathbf{r}}_{\mathrm{s}}\right)\right] \mathrm{d} s \\
& +\int_{S^{+}}\left[\rho_{A} \mathbf{v}_{A} \otimes\left(\mathbf{w}-\mathbf{v}_{A}\right) \mathbf{n}^{+}-\dot{\mathbf{r}}_{A}\right] \mathrm{d} s \\
& +\int_{S^{-}}\left[\rho_{B} \mathbf{v}_{B} \otimes\left(\mathbf{w}-\mathbf{v}_{B}\right) \mathbf{n}^{-}-\dot{\mathbf{r}}_{B}\right] \mathrm{d} s \\
& +\int_{l}\left[\rho_{\mathrm{s}} \mathbf{v}_{\mathrm{s}} \otimes\left(\mathbf{w}_{\|}-\mathbf{v}_{\mathrm{s} \|}\right) \mathbf{n}_{l}-\dot{\mathbf{r}}_{l}\right] \mathrm{d} l=0 .
\end{aligned}
$$

Three last integrals describe the rate of momentum carried by the exchange of mass between subsystems. Using (30-32) we get: 
$\dot{\mathbf{r}}_{A}=\dot{m}_{A} \mathbf{v}_{A} ; \quad \dot{\mathbf{r}}_{B}=\dot{m}_{B} \mathbf{v}_{B}$,

$\dot{\mathbf{r}}_{\mathrm{s}}=\dot{m}_{\mathrm{s}} \mathbf{v}_{\mathrm{s}}=\dot{m}_{A}\left(\mathbf{v}_{A}-\mathbf{v}_{\mathrm{s}}\right)+\dot{m}_{B}\left(\mathbf{v}_{B}-\mathbf{v}_{\mathrm{s}}\right)$,

$\dot{\mathbf{r}}_{l}=\dot{m}_{l} \mathbf{v}_{\mathrm{s}}$.

Finally, we can write the local form of the momentum balance as:

$$
\begin{aligned}
& \partial_{t}(\rho \mathbf{v})+\operatorname{div}(\rho \mathbf{v} \otimes \mathbf{v}+\mathbf{p})=\rho \mathbf{b} \text { for } \mathcal{A} \cup \mathcal{B}, \\
& \partial_{t}\left(\rho_{\mathrm{s}} \mathbf{v}_{\mathrm{s}}\right)+\operatorname{div}_{\mathrm{s}}\left(\rho_{\mathrm{s}} \mathbf{v}_{\mathrm{s}} \otimes \mathbf{v}_{\mathrm{s} \|}\right)-w_{n} \mathrm{I}_{b} \rho_{\mathrm{s}} \mathbf{v}_{\mathrm{s}} \\
& \quad+\operatorname{div}_{\mathrm{s}} \mathbf{p}_{\mathrm{s}}+\partial_{n}\left(\mathbf{p}_{\mathrm{s}} \mathbf{n}\right)+\left[\mathbf{p}_{A} \mathbf{n}_{A}+\mathbf{p}_{B} \mathbf{n}_{B}+\mathbf{f}_{S A}+\mathbf{f}_{S B}\right] \\
& =\rho_{\mathrm{s}} \mathbf{b}_{\mathrm{s}}+\dot{m}_{A}\left(\mathbf{v}_{A}-\mathbf{v}_{\mathrm{s}}\right)+\dot{m}_{B}\left(\mathbf{v}_{B}-\mathbf{v}_{\mathrm{s}}\right) \quad \text { on } \mathcal{M},
\end{aligned}
$$

Repeating now the reasoning of d'Alembert and Euler, we can define a surface d'Alembert-Euler acceleration vector to be:

$\mathbf{a}_{\mathrm{s}}=\frac{\mathrm{d}_{\mathrm{s}}}{\mathrm{d} t} \mathbf{v}_{\mathrm{s}}=\partial_{t} \mathbf{v}_{\mathrm{s}}+\left(\operatorname{grad}_{\mathrm{s}} \mathbf{v}_{\mathrm{s}}\right) \mathbf{v}_{\mathrm{s} \|} \cdot$

Using the surface identity (14), instead of divergence of the convective flux of surface momentum, we have:

$\rho_{\mathrm{s}} \mathbf{a}_{\mathrm{s}}=\partial_{t}\left(\rho_{\mathrm{s}} \mathbf{v}_{\mathrm{s}}\right)+\operatorname{div}_{\mathrm{s}}\left(\rho_{\mathrm{s}} \mathbf{v}_{\mathrm{s}} \otimes \mathbf{v}_{\mathrm{s} \|}\right)$.

The generalized Navier-Stokes layer is described now by the layer balance of mass (29) and the layer balance of momentum (42). These are two additional nonlinear differential equations for two additional fields of unknowns: the surface mass density $\rho_{\mathrm{s}}$ and the layer slip velocity $\mathbf{v}_{\mathrm{s}}$. These equations are both geometrically and physically nonlinear, and should be solved using any discretization method (FEM, FVM), assuming that the surface $\mathcal{M}$ possesses an independent from the bulk space discretization. Only if the geometrical velocity $\mathbf{w}=0$, as in the case when $\mathcal{M}^{-}$is a fixed solid surface, discretization mesh could be fixed in the marching time of numerical solution. If $\mathbf{w} \neq 0$, a moving, self deforming mesh must be resolved together with surface mass and surface momentum equations and the appropriate set of equations for bulk. In the literature, there are different cases of using the Navier-Stokes layer balances. For example, in the case when $\mathcal{A}$ and $\mathcal{B}$ are ideal, non-viscous Euler fluids, and the surface density is equal to zero $\rho_{\mathrm{s}}=0$, and the layer momentum flux is omitted $\mathbf{p}_{\mathrm{s}}=0$, then the surface mass and momentum equations reduce to the generalized form of the celebrated Rankine-Hugoniot jump conditions:

$\left\{\begin{array}{l}\dot{m}_{A}=\dot{m}_{B} \\ \dot{m}_{A} \mathbf{v}_{A}+p_{A} \mathbf{n}_{A}=\dot{m}_{B} \mathbf{v}_{B}+p_{B} \mathbf{n}_{B}\end{array}\right.$,

where $p_{A}, p_{B}$ are thermodynamic pressure in the Euler fluids $\mathcal{A}$ and $\mathcal{B}$, respectively. If, additionally $\mathbf{w}=0$, and there is an additional contribution to the surface diade $\mathbf{p}_{\mathrm{s}}=$ $\gamma \mathbf{I}_{\mathrm{s}}$, then, the layer momentum balance leads to the generalized Young-Laplace equation: $\operatorname{div}_{\mathrm{s}}\left(\gamma \mathbf{I}_{\mathrm{s}}\right)+p_{A} \mathbf{n}_{A}+p_{B} \mathbf{n}_{B}=\left[\gamma\left(\frac{1}{r_{1}}+\frac{1}{r_{2}}\right)+p_{A}-p_{B}\right] \mathbf{n}=0$.

Next, if an interfacial density is omitted $\rho_{\mathrm{s}}=0$, then, a difference between the external friction forces $\mathbf{f}_{S A}$ and $\mathbf{f}_{S B}$ vanishes, and a one common layer friction force exists:

$\mathbf{f}_{A B}=\mathbf{f}_{S A}+\mathbf{f}_{S B}=v\left(\mathbf{v}_{A}-\mathbf{v}_{B}\right)$,

where $v$ is an external viscosity coefficient. It is an exact form of external friction force proposed by Navier $\left(\mathbf{v}_{B}=0\right)$ and Stokes $\left(\mathbf{v}_{B}=\mathbf{v}_{\text {wall }}\right)$. Assuming, further, that $\mathcal{A}$ continuum is an incompressible viscous fluid: $\mathbf{p}_{A}=$ $p \mathbf{I}-2 \mu \mathbf{d}$, and $\mathcal{B}$ continuum is the rigid ${ }^{1}$, fixed solid: $\mathbf{p}_{B}=$ $0, \mathbf{v}_{B}=0$ we arrive to the Navier slip boundary condition:

$\mathbf{f}_{A B}+\mathbf{p}_{A} \mathbf{n}_{A}=v \mathbf{v}_{A}+(p \mathbf{I}-2 \mu \mathbf{d}) \mathbf{n}=0 \quad$ on $\mathcal{M}$,

where $\mathbf{v}_{\mathrm{s}}=\mathbf{v}_{A \mid \mathcal{M}}$ is identified with the slip velocity.

\section{Constitutive relations for surface momentum flux}

Let us discuss shortly the well known in the literature constitutive relations for the layer flux of momentum. In general, it is responsible for recoverable and viscous transport: $\mathbf{p}_{\mathrm{s}}=\mathbf{p}_{\mathrm{s}}{ }^{(c)}+\mathbf{p}_{\mathrm{s}}{ }^{(v)}$. The first most important part of the elastic recoverable diade $\mathbf{p}_{\mathrm{s}}^{(c)}$ known as the capillarity diade is described by the surface tension $\gamma$-the same quantity which was introduced to the process of mathematical modeling by Young, Laplace, and Poisson. The second contribution comes from the recoverable stresses called the surface bending $C_{1}, C_{2}$ and introduced by Gibbs. Another one is a layer "normal pressure" $\varpi$, introduced by Stokes (1845). These altogether lead to the following definition of the capillarity diade:

$\mathbf{p}_{\mathrm{s}}{ }^{(c)}=\varpi \mathbf{n} \otimes \mathbf{n}+\gamma \mathbf{I}_{\mathrm{s}}+C \mathbf{I I}_{\mathrm{s}}, \quad \partial_{n}\left(\mathbf{p}_{\mathrm{s}} \mathbf{n}\right)=\varpi \mathbf{n}$,

where $2 C=C_{1}+C_{2}$, and $\operatorname{div}_{\mathrm{s}} \mathbf{p}_{\mathrm{s}}^{(c)}=\gamma \mathrm{I}_{b} \mathbf{n}+C\left(\mathrm{I}_{b}^{2}-2 \mathrm{II}_{b}\right) \mathbf{n}$. Recently, a quite general form of the capillarity diade has been proposed by Badur and Karcz (2010) as:

$\mathbf{p}_{\mathrm{s}}{ }^{(c)}=\gamma_{0}-\mathbf{I}_{\mathrm{s}} \gamma_{1}+\mathbf{n} \otimes \mathbf{I}_{\mathrm{s}} \boldsymbol{d i v _ { \mathrm { s } }}\left(\gamma_{1}-\mathbf{I I}_{\mathrm{s}} \gamma_{2}\right)$,

where, for instance, the surface capillary measures can be defined to be spherical:

$\gamma_{0}=\gamma \mathbf{I}_{\mathrm{s}}, \quad \gamma_{1}=C \mathbf{I I}_{\mathrm{s}}, \quad \gamma_{2}=K \mathbf{I I I}_{\mathrm{s}}$.

\footnotetext{
${ }^{1}$ An example how to define $\mathbf{p}_{B}$ for the deformable wall is given by dell'Isola et al (2009).
} 
These are expressed in terms of the first, second, and third fundamental forms, and $\gamma, C, K$ are a surface tension, bending, and torque. However, if we suppose that the surface tension $\gamma$ is a surface dependent quantity, for instant, Bilicki and Badur (2003):

$\gamma=\sigma\left(\rho_{\mathrm{s}}-\rho_{\mathrm{s} 0}\right)$,

where $\sigma$ is a constant-like tension, $\rho_{\mathrm{s} 0}$-an equilibrium-like surface density, then we $\operatorname{got}: \operatorname{div}_{\mathrm{s}}\left(\gamma \mathbf{I}_{\mathrm{s}}\right)=\left(\operatorname{grad}_{\mathrm{s}} \gamma\right) \mathbf{I}_{\mathrm{s}}+$ $\gamma \operatorname{div}_{\mathrm{s}} \mathbf{I}_{\mathrm{s}}$. From $\operatorname{grad}_{\mathrm{s}} \gamma \neq 0$, it follows that the Marangoni ripples and other capillary effects can additionally be described (Goodrich 1981). Assuming that the rate of change $\rho_{\mathrm{s}}$ is only the result of mass flow rate coming from the fluid $\mathcal{A}$ we put:

$\dot{m}_{A}=\rho_{A} \mathbf{v}_{A} \cdot \mathbf{n}_{A}=\tau^{-1}\left(\rho_{\mathrm{s}}-\rho_{\mathrm{s} 0}\right)$,

where $\tau$-is the Bilicki-Kestin relaxation time (Bilicki and Badur 2003). Next, using the surface mass balance (29): $\operatorname{div}_{\mathrm{s}}\left(\rho_{\mathrm{s}} \mathbf{v}_{\mathrm{s}}\right)=-\tau^{-1}\left(\rho_{\mathrm{s}}-\rho_{\mathrm{s} 0}\right)$, we can find the tangential components of surface velocity $\mathbf{v}_{\mathrm{s}}$, by using a simplified, steady-state form of the momentum balance (42) as:

$$
\begin{aligned}
& \mu \mathbf{I}_{\mathrm{s}}\left(\operatorname{grad} \mathbf{v}_{A}+\operatorname{grad}^{\mathrm{T}} \mathbf{v}_{A}\right) \mathbf{n}+\operatorname{div}_{\mathrm{s}}\left(\gamma \mathbf{I}_{\mathrm{s}}\right)+v\left(\mathbf{v}_{A}-\mathbf{v}_{\mathrm{s}}\right) \\
& \quad=\dot{m}_{A} \mathbf{v}_{A},
\end{aligned}
$$

which in special cases can be expressed analytically.

The viscous properties of the Navier-Stokes layer depend on the co-called "apparent viscosity" which, in general, possesses a transversal anisotropy (Goodrich 1981). Using now the surface diade of the rate of deformation (6) and a normal change $v_{n, n}$, one can define the viscous surface stresses as:

$$
\begin{aligned}
\mathbf{p}_{\mathrm{s}}^{(v)}= & \lambda^{\prime}\left(\operatorname{trd}_{\mathrm{s}}\right) \mathbf{I}_{\mathrm{s}}+\lambda^{\prime \prime} v_{n, n} \mathbf{n} \otimes \mathbf{n}+2 \mu^{\prime} \mathbf{I}_{\mathrm{s}} \mathbf{d}_{\mathrm{s}} \mathbf{I}_{\mathrm{s}} \\
& +2 \mu^{\prime \prime}\left(\mathbf{d}_{\mathrm{s}}-\mathbf{I}_{\mathrm{s}} \mathbf{d}_{\mathrm{s}} \mathbf{I}_{\mathrm{s}}\right) .
\end{aligned}
$$

This diade does not undergo the classical 3D de SaintVenant condition, saying that the viscous stresses must be traceless. In a special case when $\lambda^{\prime \prime}=\mu^{\prime \prime}=0$ this constitutive relation was proposed by Boussinesq and also by Scriven (1960):

$\mathbf{p}_{\mathrm{s}}^{(v)}=\left(\lambda^{\prime}-\mu^{\prime}\right)\left(\operatorname{tr} \mathbf{d}_{\mathrm{s}}\right) \mathbf{I}_{\mathrm{s}}+2 \mu^{\prime} \mathbf{I}_{\mathrm{s}} \mathbf{d}_{\mathrm{s}} \mathbf{I}_{\mathrm{s}}$.

The formula for (internal) surface viscosity coefficients $\lambda^{\prime}, \mu^{\prime}$ needs extended investigations by any case, these coefficients have nothing common with the internal viscosity $\mu$ of fluid $\mathcal{A}$. Note also that another contributions to diade $\mathbf{p}_{\mathrm{s}}$, that come from additional surface fields, like the chemical potential, phase transition parameter, turbulent intermittency parameter, surface entropy and temperature, and surface electric potential, etc., are also possible, but this issue needs more elaboration.

\section{Comparison of the Navier, Stokes, Reynolds, and Maxwell slip layers}

Having the balance of slip layer momentum (42), one can easily obtain the particular cases linked to developing steps in the formation of the Navier-Stokes full set of equations. Let us note, that in the case of thin material layer, the surface density is usually omitted $\rho_{\mathrm{s}}=0$, then the inertia and body forces are equal to zero. If, additionally, the mass migration to the layer is negligible, then the balance of momentum rate turns into sum of the surface forces:

$\operatorname{div}_{\mathrm{s}} \mathbf{p}_{\mathrm{s}}+\partial_{n}\left(\mathbf{p}_{\mathrm{s}} \mathbf{n}\right)+\left[\mathbf{p}_{A} \mathbf{n}_{A}+\mathbf{p}_{B} \mathbf{n}_{B}+\mathbf{f}_{A B}\right]=0$ on $\mathcal{M}$.

Looking at this equation from the point of view of a historical development we can easily identify its particular elements in the works of pioneers and veterans of fluid mechanics.

\subsection{Navier slip boundary condition}

Firstly, omitting the surface stress $\mathbf{p}_{\mathrm{s}}=0$, and taking the body $\mathcal{B}$ as a fixed, rigid body $\mathbf{p}_{B}=0$, and the body $\mathcal{A}$ as an incompressible fluid: $\mathbf{p}_{A}=p \mathbf{I}-2 \mu \mathbf{d}$, we can formulate analytically the first in history of mechanics the slip boundary condition, originally given by Navier. Let us recall that Navier proposed the following external friction force $\mathbf{f}_{A B}=v\left(\mathbf{v}_{A}-\mathbf{v}_{\text {wall }}\right)=v \mathbf{v}_{A}$. In this case, $\mathbf{v}_{A}=\mathbf{v}_{\mathrm{s}}$ on $\mathcal{M}$ is called the velocity slip. From his own variational formulation of Molecular Dynamics Navier was able to get the following set of governing equations (Navier 1827, p. 415):

$\mathbf{f}_{\text {body }}+\operatorname{div}(-p \mathbf{I})=\rho \mathbf{a}-\operatorname{div}(2 \mu \mathbf{d}) \quad$ in $\mathcal{A}$

and boundary conditions, nowadays called "the slip boundary conditions" (Navier 1827, p. 415):

$\nu \mathbf{v}=2 \mu \mathbf{d n} \quad$ on $\quad \mathcal{M}$.

Taking into account that the wall is impenetrable by fluid i.e.v $\cdot \mathbf{n}=0$, Navier simplifies the above set of equations to the following form:

$v \mathbf{v}=\mu(\operatorname{grad} \mathbf{v}) \mathbf{n}$.

Two independent viscosity coefficients appear in this celebrated equation. The first one is an internal viscosity $\mu$ and the second one is an external viscosity $v$ ( $\varepsilon$ and $E$ in original Navier notation). Therefore, finishing his own derivations of equations of motion of viscous fluid, Navier adds comments on a dependency of the coefficient $v$ on a type of wall material (cooper, glass). Next, trying to compare the model of internally and externally viscous flow with Girard's experiments, Navier has prepared three analytical 
solutions for different flows in pipes and open channels. In his solutions both viscous coefficients $\mu$ and $v$ take a partand, what is important, for future definition of a length of slip $l_{\mathrm{s}}$, a ratio $\left[l_{\mathrm{s}}=\right] \mu / v$ frequently appears. Navier has proposed also the first closure for the slip length for water and glass as equal $l_{\mathrm{s}}=\mu /(0.0023 \rho)$, where $\mu, \rho$ are internal viscosity, and internal density of water respectively.

\subsection{Stokes slip boundary condition}

In 1845, Stokes extends Navier's model of a viscous fluid in the bulk and in the boundary layer. He proposes the following constitutive equation for the momentum flux:

$\mathbf{p}=p \mathbf{I}-2 \mu(\mathbf{d}-\delta \mathbf{I})-3 \kappa \delta \mathbf{I}=p \mathbf{I}-2 \mu \mathbf{d}+\left(\frac{2}{3} \mu-\kappa\right) \mathrm{I}_{d} \mathbf{I}$,

where two coefficients of internal viscosity appear: shear $\mu$, and bulk $\kappa$. The dilatational change of volume is described by $3 \delta=\mathrm{I}_{\mathrm{d}}=\operatorname{tr}(\mathbf{d})$. Supposing the bulk viscosity $\kappa=0$; and that the internal friction coefficient $\mu$ is homogeneous, i.e. constant in space, he gets from the momentum balance $\rho(\dot{\mathbf{v}}-\mathbf{b})+\operatorname{div} \mathbf{p}=0$ :

$$
\begin{aligned}
& \rho(\dot{\mathbf{v}}-\mathbf{b})+\operatorname{grad} p-\mu\left[\operatorname{lap} \mathbf{v}+\operatorname{div}\left(\operatorname{grad}^{\mathrm{T}} \mathbf{v}\right)\right] \\
& \quad+\frac{2}{3} \mu \operatorname{gradI}_{d}=0
\end{aligned}
$$

or within the Cartesian coordinates (Stokes 1845):

$\rho(\dot{\mathbf{v}}-\mathbf{b})+\operatorname{grad} p-\mu$ lapv $-\frac{\mu}{3} \operatorname{gradI}_{d}=0$.

In the literature, the equations in the form (63) are usually called the "Navier-Stokes equations". These equations are geometrically nonlinear with respect to the definition of the acceleration vector, and physically nonlinear with respect to the relation between pressure, density, temperature, and entropy - however, this relation has not been mentioned by Stokes explicitly. With respect to the internal friction $\mu$, these equations are linear-this is an exact prolongation of the Newtonian one-dimensional case into a threedimensional one. A simple type of nonlinearity with respect to the internal friction was postulated by Stokes, who assumed that the coefficient of viscosity, in general, should be a function of the rate of deformation $\mathbf{d}$. On the boundary slip layer, Stokes supposes a more general equation than the Navier one. He additionally takes into account a "normal pressure" $\varpi$ within the slip layer, and the tangential pressure of the layer in the form of surface tension $\gamma \mathbf{I}_{\mathrm{s}}$. He also assumes a priori known field of slip velocity $\mathbf{v}_{B}=\mathbf{v}_{\text {wall }}$. Then, his balance of layer forces can be written as a simple sum of the external forces: $\operatorname{div}_{\mathrm{s}} \mathbf{p}_{\mathrm{s}}+\mathbf{f}_{A B}+\mathbf{p}_{A} \mathbf{n}_{A}+\mathbf{p}_{B} \mathbf{n}_{B}-\varpi \mathbf{n}=0$.

In practice, because surface $\mathcal{M}^{+}$and $\mathcal{M}^{-}$are nearly parallel, we have to use only one normal vector $\mathbf{n}=\mathbf{n}^{+}=\mathbf{n}^{-}$. Then, the forces can be defined as:

- traction from fluid $\mathcal{A}$ :

$\mathbf{p}_{A} \mathbf{n}_{A}=[p \mathbf{I}-2 \mu(\mathbf{d}-\delta \mathbf{I})-3 \kappa \delta \mathbf{I}] \mathbf{n}$,

- surface tension force:

$\operatorname{div}_{\mathrm{s}} \mathbf{p}_{\mathrm{s}}=\operatorname{div}_{\mathrm{s}}\left(\gamma \mathbf{I}_{\mathrm{s}}\right)=\gamma \operatorname{tr}\left(\mathbf{I I}_{\mathrm{s}}\right) \mathbf{n}$,

- external friction force:

$\mathbf{f}_{A B}=v\left(\mathbf{v}_{A}-\mathbf{v}_{\text {wall }}\right)$,

- normal pressure of layer:

$\partial_{n}\left(\mathbf{p}_{\mathrm{s}} \mathbf{n}\right)=\varpi \mathbf{n}$,

what leads to the following Stokes slip boundary condition (1845, Sect. I, Eq. 18):

$$
\begin{aligned}
& \left(\varpi-p-\gamma \operatorname{tr} \mathbf{I I}_{\mathrm{s}}\right) \mathbf{n}+v\left(\mathbf{v}_{A}-\mathbf{v}_{\text {wall }}\right)+2 \mu \mathbf{d n} \mathbf{n} \\
& \quad-2 \mu \delta \mathbf{n}+3 \kappa \delta \mathbf{n}=0 .
\end{aligned}
$$

It turns out that the friction force $\mathbf{f}_{A B}$ is known only after solution of the problem, whereas in the case of a free surface this force is known as a given data. There remain to consider the case of two fluids having a common surface given by $\mathcal{M}=0$. Fluid velocities are $\mathbf{v}_{A} \equiv \mathbf{v}\left(\right.$ side $\left.\mathcal{M}^{+}\right)$ and $\mathbf{v}_{B} \equiv \mathbf{v}^{\prime}$ (side $\mathcal{M}^{-}$). In such a case it is important to put the condition of impermeability, saying that the normal velocities should be the same:

$$
\begin{aligned}
& \mathbf{v} \cdot \mathbf{n}+\mathbf{v}^{\prime} \cdot \mathbf{n}^{\prime}=0 \quad \text { or } \\
& l\left(u-u^{\prime}\right)+m\left(v-v^{\prime}\right)+n\left(w-w^{\prime}\right)=0 .
\end{aligned}
$$

Let the interface layer $\mathcal{M}^{+} \mathcal{M}^{-}$between both fluids possesses a Navier-like friction force, due to postulated by Rybczyński (1911) an external viscosity, say $v_{A B}$, which in general, differs from the external viscosity between a fluid and a surface. Then, in analogy to the previous consideration, we have a following set of governing equations (Stokes 1845, Sect. I, Eq. 17):

$\mathbf{f}_{A B}+\mathbf{p}_{A} \mathbf{n}_{A}+\mathbf{p}_{B} \mathbf{n}_{B}=0$

where an external friction force within the layer is:

$\mathbf{f}_{A B}=v_{A B}\left(\mathbf{v}_{A}-\mathbf{v}_{B}\right)$,

and two external traction forces on the surfaces bounding the layer

$\mathbf{p}_{A} \mathbf{n}_{A} \equiv \mathbf{p n}=[p \mathbf{I}-2 \mu(\mathbf{d}-\delta \mathbf{I})-3 \kappa \delta \mathbf{I}] \mathbf{n}$,

$\mathbf{p}_{B} \mathbf{n}_{B} \equiv \mathbf{p}^{\prime} \mathbf{n}^{\prime}=\left[p^{\prime} \mathbf{I}-2 \mu^{\prime}\left(\mathbf{d}^{\prime}-\delta^{\prime} \mathbf{I}\right)-3 \kappa^{\prime} \delta^{\prime} \mathbf{I}\right] \mathbf{n}^{\prime}$.

Suppose $v_{A B} \rightarrow 0$, as appears most probable, the problem significantly simplifies. Nevertheless, we are not sure under 
which circumstances the external viscosity between two different fluids can be measured, and when its appearance is essentially important.

\subsection{Reynolds slip boundary condition}

In 1879, standing on the ground of Kinetic Theory arguments, Reynolds has proposed the following set of momentum balance within the rarified gas:

- the pressure tensor in the bulk:

$\mathbf{p}_{A}=p \mathbf{I}-2 \mu \mathbf{d}+\frac{2}{3} \mu \mathrm{I}_{d} \mathbf{I}+2 \beta \mathbf{d}_{(2)}$,

- momentum balance in the bulk:

$\rho \mathbf{a}+\beta$ lapa $+\operatorname{grad} p-\mu$ lapv $-\frac{1}{3} \mu \operatorname{gradI}_{d}=\rho \mathbf{b}$,

- momentum balance at the boundary layer:

$-\varpi \mathbf{n}+\mathbf{f}_{A B}+\mathbf{p}_{A} \mathbf{n}_{A}=0$.

The coefficient $\beta=r / p$ is inversely proportional to pressure. Here, Reynolds proposes a new definition of the Cauchy momentum flux, where an acceleration of deformation diade, say $\mathbf{d}_{(2)}$, defined as a symmetric tensor, appears:

$\mathbf{d}_{(2)}=\frac{1}{2}\left(\operatorname{grad} \mathbf{a}+\operatorname{grad}^{\mathrm{T}} \mathbf{a}\right) \neq \dot{\mathbf{d}}$,

with acceleration defined to be: $\mathbf{a}=\partial_{t} \mathbf{v}+(\operatorname{grad} \mathbf{v}) \mathbf{v}$.

Let us now return, to the definition of external friction force $\mathbf{f}_{A B}$, given by Reynolds (1879). Here, the first time in the literature, a contribution from transpiration phenomena has appeared. But independently from the thermal transpiration discovery, a great Reynolds' scientific goal was a unification of three phenomena: the Graham pressure transpiration, the Graham concentration transpiration, and just discovered by Reynolds, the thermal transpiration into a one generalized model of transpiration phenomena. In order to show how Reynolds do this, let, according to Reynolds' denotations, $\varpi, \theta, N$ means the normal pressure, temperature, and mole concentration of a gaseous component (for instance $N_{1}=\rho_{1} / M_{1}$ ), respectively. These parameters are defined in the slip layer. We can obtain a unified model of pressure, temperature, and concentration transpirations in the boundary layer, by adding the particular contributions described by equations (see Reynolds 1879, Eqs. 110, 112, and 117):

$\mathbf{f}_{A B}=v\left(\mathbf{v}-\mathbf{v}_{\text {wall }}-c_{v \varpi} \operatorname{grad}_{\mathrm{s}} \varpi-c_{v \theta} \operatorname{grad}_{\mathrm{s}} \theta-c_{v N} \operatorname{grad}_{\mathrm{s}} N\right)$.

Here, one can see, three serious differences with celebrated Maxwell's boundary condition (85). The first one, is an explicit appearance of the pressure $\operatorname{gradient} \operatorname{grad}_{\mathrm{s}} \varpi$, realizing the Graham pressure transpiration phenomena, called sometimes the pressure driven motion. The pressure mobility coefficient $c_{v \varpi}$ is different for different gases for the same material of capillary tube. For instance, it was found by Graham $(1846,1849)$ that the ratio of $c_{v \sigma}$ for air and hydrogen is 2.04 . The second difference concerns more correct definition of the thermal mobility coefficient $c_{\nu \theta}$ than Maxwell has made. For description of the thermal mobility Reynolds has introduced not one, but four accommodation coefficients $f_{1}, f_{2}, f_{3}, f_{4}$. Two first depend, separately, on the properties of solid surface material and a kind of gas. The next two describe a more complete than Maxwell's, process of interaction of a gas with a boundary. The third difference is related to the Graham diffusional transpiration described by the concentration mobility coefficient $c_{v N}$. These three differences allow us to say that there is a substantial base for distinction between both models of boundary layer. Following Stokes boundary layer equation: $-\varpi \mathbf{n}+\mathbf{f}_{A B}+$ $\mathbf{p}_{A} \mathbf{n}_{A}=0$, such a model can be presented shortly to be:

$$
\begin{aligned}
& v\left(\mathbf{v}-\mathbf{v}_{\text {wall }}-c_{v \varpi} \operatorname{grad}_{\mathrm{s}} \varpi-c_{v \theta} \operatorname{grad}_{\mathrm{s}} \theta-c_{v N} \operatorname{grad}_{\mathrm{s}} N\right) \\
& \quad+(p-\varpi) \mathbf{n}-2 \mu \mathbf{d n}+2 \beta \mathbf{d}_{(2)} \mathbf{n}=0 .
\end{aligned}
$$

\subsection{Maxwell slip boundary condition}

Also in 1879, Maxwell standing on the ground of the nonequilibrium version of his Kinetic Theory of gases has proposed the following set of equations:

- the pressure tensor:

$$
\begin{aligned}
\mathbf{p}= & p \mathbf{I}-2 \mu \mathbf{d}+\frac{2}{3} \mu \mathbf{I}_{d} \mathbf{I}+\beta_{1} \frac{1}{2}\left(\operatorname{grad} \mathbf{g}+\operatorname{grad}^{\mathrm{T}} \mathbf{g}\right) \\
& +\beta_{2}(\operatorname{divg}) \mathbf{I},
\end{aligned}
$$

- momentum balance in the bulk:

$$
\begin{gathered}
\rho \dot{\mathbf{v}} \\
+\operatorname{grad} p-\mu \operatorname{lap} \mathbf{v}-\frac{1}{3} \mu \operatorname{gradI}_{d} \\
+\left(\beta_{1}+\beta_{2}\right) \operatorname{grad}(\operatorname{lap} \theta)=\rho \mathbf{b} .
\end{gathered}
$$

Here, according to the nonequilibrium kinetic treatment, Maxwell was able to estimate the value of the thermal transpirations constants to be:

$\beta_{1}=3 \frac{\mu^{2}}{\rho \theta} ; \quad \beta_{2}=\frac{3}{2} \frac{\mu^{2}}{\rho \theta}$.

Let us note, that the pressure tensor contains only a linear contribution from the gradient of temperature $\operatorname{grad} \mathbf{g}=$ $\operatorname{grad}^{\mathrm{T}} \mathbf{g}=\theta, i j \mathbf{e}_{i} \otimes \mathbf{e}_{j} . \quad$ But exploring Natanson's fully nonlinear version of the Kinetic Theory, one can find also the second order contribution (Badur and Sun 1995):

$$
\begin{aligned}
\mathbf{p}= & p \mathbf{I}-2 \mu \mathbf{d}+\frac{2}{3} \mu \mathbf{I}_{d} \mathbf{I}+\beta_{1} \frac{1}{2}\left(\operatorname{grad} \mathbf{g}+\operatorname{grad}^{\mathrm{T}} \mathbf{g}\right) \\
& +\beta_{2}(\operatorname{divg}) \mathbf{I}+\beta_{3} \mathbf{g} \otimes \mathbf{g} .
\end{aligned}
$$


It should be noted, however, that boundary condition, where the coefficient $\beta_{3}$ and the first gradient of temperature $\mathbf{g}$ appear, is fundamentally quite different from original Maxwell's one, since $\mathbf{g}$ appears within the friction force $\mathbf{f}_{A B}$ what physically means different phenomena. It is also a historical truth that the presence of $\mathbf{g}$ in the boundary layer was firstly postulated by Reynolds, and next proven by Maxwell.

Turning to the Maxwell slip boundary layer in an original formulation, let us suppose that the surface is a plane $y, z$ and the gas flows in that side of a plane for which $x$ is positive. Let $v$ be a main surface component of velocity in the main direction $y$, then the slip-velocity formula is given by the following expression (Maxwell 1879, Appendix Eq. 68):

$v-G\left(\frac{\mathrm{d} v}{\mathrm{~d} x}-\frac{3}{2} \frac{\mu}{\rho \theta} \frac{\mathrm{d}^{2} \theta}{\mathrm{d} x \mathrm{~d} y}\right)-\frac{3}{4} \frac{\mu}{\rho \theta} \frac{\mathrm{d} \theta}{\mathrm{d} y}=0$,

where $G$ is the Helmholtz-Piotrowski slip length, $\mu-$ internal viscosity coefficient, $\rho$-gas density, $\theta$-absolute temperature. To finish the process of reconstruction of Maxwell slip boundary condition (85), let us write the boundary force condition in terms of the Navier-Stokes layer in a general form:

$\mathbf{f}_{A B}+\mathbf{p}_{A} \mathbf{n}_{A}+\mathbf{p}_{B} \mathbf{n}_{B}=0$,

where

- the fluid boundary force:

$$
\begin{aligned}
\mathbf{p}_{A} \mathbf{n}_{A}= & {\left[p \mathbf{I}-2 \mu \mathbf{d}+\frac{2}{3} \mu \mathrm{I}_{d} \mathbf{I}+\beta_{1} \frac{1}{2}\right.} \\
& \left.\times\left(\operatorname{grad} \mathbf{g}+\operatorname{grad}^{\mathrm{T}} \mathbf{g}\right)+\beta_{2}(\operatorname{div} \mathbf{g}) \mathbf{I}\right] \mathbf{n},
\end{aligned}
$$

- the rigid body surface boundary:

$\mathbf{p}_{B} \mathbf{n}_{B}=0$,

- the surface friction force:

$\mathbf{f}_{A B}=v\left(\mathbf{v}-\mathbf{v}_{\text {wall }}-c_{v \theta} \operatorname{grad}_{\mathrm{s}} \theta\right)$.

According to Maxwells formula (85), the thermomobility coefficient $c_{v \theta}$ should be stated as a coefficient that is not dependent on the property of the solid surface:

$c_{v \theta}=\frac{3}{4} \frac{\mu}{\rho \theta}$.

Next, using the definition $l_{\mathrm{s}}=G=\mu / v$, and after dividing the balance (86) by the external viscosity $v$ we obtain a generalization of the Maxwell slip boundary layer as:

$$
\begin{gathered}
\mathbf{v}-\mathbf{v}_{\text {wall }}-c_{v \theta} \operatorname{grad}_{\mathrm{s}} \theta+\frac{p}{v} \mathbf{n}-2 l_{\mathrm{s}} \mathbf{d n}+\frac{2}{3} l_{\mathrm{s}} \mathrm{I}_{\mathrm{d}} \mathbf{n} \\
+\frac{\beta_{1}}{v} \frac{1}{2}\left(\operatorname{grad} \mathbf{g}+\operatorname{grad}^{\mathrm{T}} \mathbf{g}\right) \mathbf{n}+\frac{\beta_{2}}{v}(\operatorname{div} \mathbf{g}) \mathbf{n} .
\end{gathered}
$$

In an analogy, the ratios $l_{\mathrm{s} \beta_{1}}=\beta_{1} / v$ and $l_{\mathrm{s} \beta_{2}}=\beta_{2} / v$ can be called the thermal transpiration slip coefficients. We can conclude then, that despite $l_{s}=\mu / v$ is a main characteristic of the external viscosity, it cannot be simply treated as only one characteristic of the Navier-Stokes layer. In particular case, when the rigid cold particle is immersed into a gas at rest $(\mathbf{v} \equiv 0)$, and assuming the linearity of the temperature distribution ( $\operatorname{gradg}=0$ ), we obtain the thermal velocity of a particle:

$\mathbf{U}=\mathbf{v}_{\text {wall }}=-c_{\nu \theta} \operatorname{grad}_{\mathrm{s}} \theta$.

The velocity $\mathbf{U}$ is nowadays called the thermophoretic velocity (Brenner 2005). It characterizes the motion of nanoparticles that follows from the surface gradient of temperature. It is important to note, that using apparatus of the Kinetic Theory, Maxwell was able to find an explicit formula for the length of slip (Maxwell 1879, Appendix Eq. 67):

$l_{\mathrm{s}}=\frac{\mu}{v}=\frac{1}{2} \mu(2 \pi)^{\frac{1}{2}}\left(\frac{p}{\rho}\right)^{-\frac{1}{2}}\left(\frac{2}{f}-1\right)=\frac{2}{3}\left(\frac{2}{f}-1\right) l$,

where $l$ means the Meyer relation for the mean-free path of a gas molecule and $f$ being the fraction absorbed. When $f=1 / 2$, or the surface acts as if it were half perfectly reflecting and half perfectly absorbing, then we got $l_{\mathrm{s}}=2 l$. If it were wholly absorbent, then $l_{\mathrm{s}}=2 / 3 l$. In practice, the slip length depends on a kind of surface material and gasfor instance, from Knudt and Warburg experimental data it follows that for air on a glass surface in $17^{\circ} \mathrm{C}$ is $l_{\mathrm{s}}=8 / p$, and for hydrogen is $l_{\mathrm{s}}=15 / p$, where the pressure is given in dynes per square centimeter. In the modern literature, the coefficient of a partial absorption $f$ is usually called "tangential momentum accommodation coefficient" (TMAC). This coefficient is not dependent on heat flow, and it accounts for the average tangential to a surface momentum exchange between the fluid molecules and the solid molecules. Its value should be evaluated experimentally, but it is known that it varies from zero (for specular reflection) up to unity (for complete or diffuse accommodation) (GadEl-Hak 2001; Priezjev 2007).

\section{Conclusions}

In this article, the flow enhancement phenomena in microand nano-channels are classified within unified model, which bases on the extended Navier-Stokes boundary layer equations and includes different surface mobility mechanisms.

The Navier's boundary friction concept, which leads to the slip velocity condition, is usually considered in literature without any linkage to other surface phenomena 
(Thompson and Trojan 1997; Zhu and Granick 2001; Priezjev 2007). There are some generalizations of the NavierStokes slip boundary in literature (Karniadakis et al. 2005; Lauga and Stone 2003). These strictly follow the Maxwell line of reasoning, and introduce higher derivatives and nonlinearities. Unfortunately, these second order slip models are quite inconsistent with the bulk model, where only the first velocity gradient appears, therefore, they cannot be solved within the framework of continuum model.

In the present article, a new generalization of the Navier-Stokes boundary slip layer is proposed. The advantage of the current work is a comprehensive treatment of different phenomena on the basis of a single model. The original Navier-Stokes model is supplemented by additional surface quantities as the surface mass and the surface momentum flux. The slip velocity $\mathbf{v}_{\mathrm{s}}$ can be determined from the solution of the momentum balance (42) within a thin shell-like domain. It should be underlined that the stress tensors $\mathbf{p}_{A}$ and $\mathbf{p}_{B}$ are determined in the bulk and cannot be arbitrarily changed at the walls. The present approach provides separation of constitutive relations imposed for fulfillment of the surface momentum balance independently for bulk and wall. The modeling of the surface momentum diade $\mathbf{p}_{\mathrm{s}}$, and the surface friction force $\mathbf{f}_{A B}$, is still an open question. It is a most important fact that follows from a generalized model of the NavierStokes slip layer.

Open Access This article is distributed under the terms of the Creative Commons Attribution Noncommercial License which permits any noncommercial use, distribution, and reproduction in any medium, provided the original author(s) and source are credited.

\section{References}

Badur J, Karcz M (2010) On evolution of the notion of a capillarity tensor. In: Pietraszkiewicz W, Kreja I (eds) Shell structures: theory and applications, vol 2. CRC Press, Taylor \& Francis Group, London, pp 159-162

Badur J, Sun BH (1995) Some remarks on the structure of evolution equations. Appl Math Mech 16:747-757

Bilicki Z, Badur J (2003) A thermodynamically consistent relaxation model for turbulent binary mixture undergoing phase transition. J Non Equilib Thermodyn 28:311-340

Brenner H (2005) Navier-Stokes revisited. Physica A 349:60-132

dell'Isola F, Kosiński W (1993) Deduction of thermodynamic balance laws for bidimensional nonmaterial directed continua modelling interphase layers. Arch Mech 45:333-359
dell'Isola F, Madeo A, Seppecher P (2009) Boundary conditions at fluid-permeable interfaces in porous media: a variational approach. Int J Solids Struct 46:3150-3164

Fried E, Gurtin M (2005) Tractions, balances and boundary conditions for nonsimple materials with application to liquid flow at small-length scales. Arch Ration Mech Anal 182:513-554

Gad-El-Hak M (2001) Handbook of MEMS. CRC Press/Taylor \& Francis Group, Boca Raton/London

Goodrich F (1981) Surface viscosity as a capillary excess transport property. In: Goodrich F, Rusanov A (eds) The modern theory of capillarity. Akademie-Verlag, Berlin, pp 19-34

Graham T (1846) On the motion of gases I. Philos Trans R Soc Lond 131:573-632

Graham T (1849) On the motion of gases II. Philos Trans R Soc Lond 137:349-362

Ho C, Tai Y (1998) Micro-electro-mechanical-systems (MEMS) and fluid flow. Ann Rev Fluid Mech 30:579-612

Karniadakis GE, Beskok A, Aluru N (2005) Microflows and nanoflows: fundamentals and simulation. Springer, Berlin

Lauga E, Stone H (2003) Effective slip in pressure-driven Stokes flow. J Fluid Mech 489:55-77

Matthews M, Hill J (2009) On three simple experiments to determine slip lengths. Microfluid Nanofluid 6:611-619

Maxwell J (1879) On stresses in rarified gases arising from inequalities of temperature. Philos Trans R Soc Lond 170:231-256

Navier CLMH (1827) Mémoire sur les lois du mouvement des fluides-presented in (1822). Mémoires l'Acad Royale des Sciences de l'Institut de France 2:375-393

Pietraszkiewicz W (1977) Introduction to the non-linear theory of shells. Mitt Inst Mech Ruhr Univ Bochum 10

Priezjev N (2007) Rate-dependent slip boundary conditions for simple fluids. Phys Rev E 75:051605

Reynolds O (1879) On certain dimensional properties of matter in the gaseous state. Philos Trans R Soc Lond 170:727845

Rybczyński W (1911) Über die fortschreitende bewegung einer flüssigen kugel in einem zähen medium. Bull Int Acad Sci Cracovie A 40-46

Scriven L (1960) Dynamics of fluid interfaces. Chem Eng Sci 12:98-108

Slattery J, Sagis L, Oh ES (2007) Interfacial transport phenomena, 2nd edn. Springer, Berlin

Stokes G (1845) On the theories of the internal friction of fluids in motion, and of the equilibrium and motion of elastic solids. Trans Cambridge Philos Soc 8:287-319

Stumpf H, Badur J (1993) On objective surface rate. Q Appl Math $51: 161-181$

Thompson P, Trojan S (1997) A general boundary condition for liquid flow at solid surface. Nature 389:360-362

To Q, Bercegeay C, Lauriat G, Léonard C, Bonnet G (2010) A slip model for micro/nano gas flows induced by body forces. Microfluid Nanofluid 8:417-422

You XY, Zheng JR, Jing Q (2007) Effects of boundary slip and apparent viscosity on the stability of microchannel flow. Forschung im Ingenieurwesen 71:99-106

Zhu Y, Granick S (2001) Rate-dependent slip of newtonian liquid at smooth surfaces. Phys Rev Lett 87:096105

Zmitrowicz A (1987) A thermodynamical model of contact, friction and wear. Wear 114:135-168; 169-197; 199-221 